\title{
Toward a General Factor of Disruptiveness: The Most Novel Creative Objects Tend to Be the Least Valuable and Feasible Ones
}

\author{
Nicolas Pichot ${ }^{1,3}$, Eric Bonetto ${ }^{1,2,3}$, Thomas Arciszewski ${ }^{1,3}$, Nathalie Bonnardel ${ }^{1,3}$ \\ and Jean-Baptiste Pavani ${ }^{1}$ \\ ${ }^{1}$ PsyCLE, Aix-Marseille University, Aix-en-Provence, France \\ ${ }^{2}$ LPS, Aix-Marseille University, Aix-en-Provence, France \\ ${ }^{3}$ Aix-Marseille Institute of Creativity and Innovation (InCIAM), Aix-Marseille University, Aix-en- \\ Provence, France
}

\section{Author notes}

We have no known conflict of interest to disclose.

Nicolas Pichot is affiliated with the PsyCLE research center, Aix-Marseille University.

Correspondence concerning this article should be addressed to Nicolas Pichot, Centre PSYCLE, Aix-Marseille Université, Maison de la Recherche, 29 Avenue Robert Schuman, 13621 Aix-en-Provence Cedex 01, France. Email: nicolas.pichot@univ-amu.fr

Link Open Science Framework (OSF) data :

https://osf.io/4fa3a/?view only=92328dab6c1b4b398befba38aeb43f8b

[This paper is actually under review. This is a revised version of the submitted paper] 


\begin{abstract}
Talking about creative productions seems to be a common activity in both everyday language and the language used by researchers. The use of the term creative implies the existence of a creativity variable that allows for comparisons between different productions. According to the standard definition of creativity (Runco \& Jaeger, 2012), to be creative, a production must have both value and novelty. So far, empirical psychometric studies looking for a creativity variable with these two dimensions have shown that value and novelty are not only independent, but are also only weakly correlated. This empirical evidence, which has been widely replicated in the literature, indicates that, according to psychometric rules, it is impossible and indeed paradoxical to talk about the creativity of a production. In the present study, we sought to replicate these results by including a new dimension that has mostly been omitted in psychometric studies of creativity dimensions, namely feasibility. Results $(N=662$ ideas) tended to show that this new dimension, negatively correlated with novelty and positively correlated with value, led to a second-order general factor of creativity. We named the axis formed by these three dimensions disruptiveness in order to underline the subtle difference from what would be an axis of creativity. The theoretical and applied implications of these results are discussed.
\end{abstract}

Keywords: disruptiveness, general creativity score, assessment, production, psychometric 


\section{Psychometric Approach in the Field of Creativity}

In a chapter entitled "Psychometric approaches to the study of human creativity", published in Sternberg (1999)'s Handbook of Creativity, Plucker and Renzulli provided a comprehensive overview of the use of the psychometric approach in creativity research. The authors emphasized that this approach is not singular, but plural. This plurality seems to parallel the variety of topics addressed in creativity research. These topics can be divided into different types. The first proposal for structuring the scientific literature on creativity, known as the 4 P's of creativity, was put forward by Rhodes (1961). This author highlighted four main lines of study pursued by researchers: Person, Process, Product and Press (i.e., context). Although other proposals for structuring the literature have been put forward since then, namely Glăveanu (2013)'s 5 A's and Lubart (2017)'s 7 C's, the plurality of psychometric approaches by Plucker and Renzulli (1999) is more in line with Rhodes' model (1961), in that psychometric approaches are mainly used in the four main lines of study it identifies.

Apart from proposing a parsimonious classification of creativity research, Rhodes (1961)'s 4 P's model is interesting because it proposes a logical and hierarchical sequence of its four components. The context (press) tends to influence the creator (person). The creator is the one who sets up the process. The process leads to the creation (i.e., the creative object or product). This sequence suggests that the creative nature of the context, person and process can only be objectively assessed if the product's creative nature is not flawed (i.e., a creative context is a context leading to creative products, while a creative person is a person generating creative products). Given that there are still conflicting views on the assessment of the creative nature of products, any conclusions about the components placed higher up in the hierarchy of the 4 P's model are liable to be questioned. Plucker and Renzulli (1999, p. 90) summarized this point very well when they addressed the issue of the psychometric approach to creative productions: "MacKinnon (1978) argued that 'the starting point, indeed the bedrock of all studies of 
creativity, is an analysis of creative products, a determination of what it is that makes them different from more mundane products'" (p. 187).

\section{How to Assess Product Creativity? The Criterion Problem}

The issue of which criteria to use to define the creativity of a product, although relatively old and known as the criterion problem, is still very much alive (Cardoso de Sousa, 2008; Harrington, 2018; Plucker \& Renzulli, 1999; Weisberg, 2015, 2018). Numerous efforts to validate constructs have been carried out in recent years by researchers, with the aim of proposing criteria that reflect the concept of creativity. These efforts rely on two radically different approaches: the semantic-logical approach and the psychometric approach. The main purpose of any definition or categorization is to specify the necessary and sufficient criteria related to a given concept. The semantic-logical approach pursues this goal by relying mainly on our understanding of words, concepts, and their relationships. Within this approach, these different elements are associated with each other on the basis of logical reasoning. This method does not use any data or statistical methods to corroborate or refute the conjectures to which it gives rise. Based on this approach, the so-called standard definition of creativity proposes two criteria for qualifying the creativity of a product: originality and effectiveness (Runco \& Jaeger, 2012).

The use of these criteria was, however, recently called into question by Weisberg $(2015$, 2018), with his intentional novelty definition. Also relying on semantic-logical arguments, Weisberg $(2015,2018)$ mainly questioned the criterion of effectiveness (what he calls value ${ }^{1}$ ), suggesting that it should be replaced with a criterion of intentionality. The value criterion is described in the standard definition as a necessary criterion, in that it makes it possible to

\footnotetext{
${ }^{1}$ Other authors, such as Kaufman and Sternberg (2010), also use the term value as a generic term that is sufficiently broad to capture a variety of specific concepts, such as effectiveness, utility, appropriateness or relevance.
} 
exclude productions that are considered new but bizarre (e.g., the so-called monkey typing at the typewriter) and which, for some authors, cannot be classified as creative. The definition of the intentionality criterion counteracts this idea, by indicating that another way of distinguishing the creative from the bizarre would be to rely on the creator's intentionality. Albeit interesting, semantic-logical arguments sometimes seem hard to confirm or refute solely through logical reasoning. This is the reason why we focus on the psychometric approach in the rest of this article.

\section{Establishing the Criteria for Creativity}

The main difficulty with the supposedly most important dimensions of product creativity is that these dimensions are often named in a very generic way and include terms that are often presented as synonyms (e.g., the novelty dimension refers to criteria such as originality, unusualness, and uniqueness, while the value dimension refers to criteria such as effectiveness, utility, relevance, and appropriateness). Although this has some advantages (i.e., allowing creative products to be described according to numerous characteristics), it also has some disadvantages. In particular, frontiers between conceptually close concepts (i.e., a dimension and one of its supposed criteria such as value and effectiveness, or two criteria associated with the same dimension, such as effectiveness and utility) are blurred, and tend to trigger disagreements among researchers.

For example, Sarkar and Chakrabarti (2011) pointed out that the unusualness criterion (i.e., less frequent productions) that is often associated with the novelty dimension may provide an indirect measure of novelty, but does not necessarily represent the same concept. Another such example is the inclusion we sometimes observe in the value dimension of criteria that could potentially refer to other independent dimensions. This is particularly true for feasibilityrelated characteristics. For some researchers, value and feasibility represent the same construct. For instance, Rietzschel, Nijstad, and Stoebe (2010, p. 48) suggested that "[...] the usual 
definition of a 'good' idea is one that is both highly original (and unusual) and highly feasible (or useful)". By contrast, other researchers explicitly make this distinction, by proposing two distinct value and feasibility dimensions (e.g., Dean et al., 2006; Kudrowitz \& Wallace, 2013; MacCrimmon \& Wagner, 1994). It seems fairly easy to propose a topic that emphasizes the distinction between these two elements and shows that a production can be useful and/or efficiently meet an instruction without being feasible. For instance, the idea of using a device to teleport oneself is highly valuable in response to the instruction "generate ideas to reduce road traffic", but scarcely feasible given the current state of knowledge.

Thus, if the criteria for creativity are used to describe a product, and if we are to be able to distinguish between these criteria as clearly as possible, it seems necessary to adopt a definition of each one that (1) does not refer to synonyms, and (2) makes it possible to highlight what the criteria have in common and what tells them apart.

Novelty can be defined as the number or extent of variations present in a product in relation to what already exists in some domain at a given time. As Csikszentmihályi and Wolfe (2014, p. 162) state, "Creativity cannot exist in a vacuum; new is relative to old. Without norms there can be no variation; without standards there can be no excellence". This dimension is often assessed using items such as new, unusual, or surprising. This type of measurement refers to a subjective approximation of the reality of the gap between the product of interest and what already exists in the domain (i.e., this approximation is all the more realistic if the person assessing the product has expertise in the domain under consideration).

The value dimension is often expressed in different terms that are more or less similar (e.g., useful, efficient, effective, suitable, significant, relevant, or adapted). Although it is sometimes confused with feasibility (Rietzschel et al., 2010), and is actually correlated with it (Dean et al., 2006; Kudrowitz \& Wallace, 2013), it can nonetheless be distinguished from it. Value can be defined as the level of adequacy of the object or product in relation to the 
objectives or function it is supposed to fulfill. Just as novelty cannot exist ex nihilo, so value can only be counted in terms of the goals/objectives the product is designed to meet (Moran, 2010). Importantly, goals to achieve in a given problem can correspond to constraints regarded as mandatory (or nonpermissive). For a product to have value, it must tend to meet the goals expressed by the problem. In some cases, value may be difficult to determine objectively, and this refers to the question of well or ill-defined problems (Eastman, 1969; Simon, 1973).

The example of the well-known Phillip Stark's Juicy Salif Juicer, which deliberately represents an aesthetically pleasing production, but whose effectiveness is undermined by a lack of stability (i.e., the device is placed on three legs). Without any information other than the name and representation of the production, the question of this production's value may divide the judges (experts or not), with some choosing to lend more importance to the aesthetic aspect and artistic character of the work, and others choosing to consider this production as having no value, in that it does not fulfill or only badly fulfills the function (i.e., objectives or goals) it seems to assume (i.e., being a juicer). Thus, the value dimension and the criteria that make it up are likely to vary according to the way in which the objectives, instructions or any other mandatory constraints from the problem are presented.

A potential third dimension of creativity relating to feasibility may or may not already have been considered- The reason for this may be linked to the distinction between a creative idea and a creative product ${ }^{2}$. As Rhodes (1961) intimated, an idea is a thought that can be communicated in various formats (i.e., words, paint, etc.). More importantly, when an idea becomes embodied into tangible form it is called a product (p. 309). The common points between the psychometric studies that did not consider feasibility as a possible characteristic of creative objects is that they used products that already existed. By contrast, the outputs to be

\footnotetext{
${ }^{2}$ Other reasons can also be raised. In the field of art ( $8.30 \%$ of the studies considered here) the question of the feasibility of a production or an idea seems at first sight to have little meaning.
} 
evaluated in Dean et al. (2006)'s study were obtained following a brainstorming activity. Thus, they were ideas and lent themselves to an assessment of their feasibility. The same is true for studies that did not adopt the psychometric approach to object creativity. The feasibility criterion is only used when the outputs comes under the heading ideas (Diehl \& Stroebe, 1991; Di Mascio, Kalyuga \& Sweller, 2018; Lonergan, Scott \& Mumford, 2004; Potter \& Balthazard, 2004). Nevertheless, this dimension seems fundamental when talking about creativity. When creativity is presented as one of the key skills in the 21st century (Qian, Plucker, \& Yang, 2019; OECD, 2018) it is mainly because it refers to ideas for future products or knowledge that do not yet exist. Consequently, whereas objects that do not yet exist can be assessed on their novelty, value, and feasibility, existing products can only be assessed on their novelty and value.

The criterion of feasibility is defined in the literature as follows: "An idea is feasible if it does not violate known constraints or if it can be easily implemented" (MacCrimmon \& Wagner, 1994, in Dean et al., 2006, p. 661). Diehle and Stroebe (1987; in Dean et al., 2006, p. 661) speak of "precision and ease of implementation within available constraints". Thus, what mainly seems to emerge from these definitions is the notion of constraint, which is also present in the value dimension of creativity. Nevertheless, distinctions can be made between valuerelated constraints and feasibility-related constraints. Janssen, Jégou, Nougier, and Vilarem (1989) highlighted two types of constraint. First, validity constraints refer to goals that must imperatively be attained by the proposed solutions. They are often highlighted in the initial description of the problem. For instance, the problem statement Find solutions to improve the means of communication between individuals by taking their preferences into account contains two validity constraints, namely (1) improving means of communications between individuals, and (2) considering their preferences. Second, preference constraints represent goals that are not specified in the problem statement, but which are present implicitly and can be perceived of as more or less important by different individuals. To continue with the example of the 
problem: "Find solutions to improve the means of communication between individuals by taking their preferences into account", some individuals may be inclined to pursue goals that are not specified in these instructions (e.g., finding solutions that are not economically costly). These preference constraints are therefore more flexible than the validity constraints. We suggest that the value of an object depends on its tendency to fulfill validity constraints (i.e., nonpermissive constraints) present in the problem and identified in the problem statement, whereas the feasibility of an idea could depend on its ability to fulfill preference constraints (i.e., permissive constraints) present in the problem but not made explicit ${ }^{3}$.

\section{The Psychometric Approach Applied to Understand Product Creativity: Empirical}

\section{Results}

As we stated earlier, some authors have used the psychometric approach to study the construct validity of product creativity. Our review of the literature allowed us to collect a small number of articles $(\mathrm{N}=12)$ that specifically explored the main characteristics of product creativity, through the use of psychometric statistical tools such as exploratory factor analysis (EFA), confirmatory factor analysis (CFA) or principal component analysis (PCA) (Amabile, 1979; Besemer, 1998; Besemer \& O'Quin, 1986, 1999; Christiaans, 2002; Dean, Hender, Rodgers, \& Santanen, 2006; Demirkan \& Afacan, 2012; Form, Aue, \& Kaernbach, 2019; Horn \& Salvendy, 2006b, 2009; O'Quin \& Besemer, 1989; White \& Smith 2001). In addition to this limited number of studies, many inconsistencies can be observed from one study to another, making it difficult to derive general conclusions from their results (i.e., differences in fields of study, theoretical models, number and type of evaluators, etc.).

\footnotetext{
${ }^{3}$ However, there is a special case where this idea is wrong. In a problem where the instructions would explicitly require that the idea produced be feasible then the feasibility would be a validity constraint and be loading in the value dimension
} 
Despite all their limits, it is noteworthy that they all analyze at least two criteria: novelty and value. Moreover, the results on these criteria tend to be replicated from one study to another. More specifically, items developed to assess novelty-related characteristics group together to form one dimension of product creativity, items developed to assess value-related characteristics group together to form a second dimension, and these two novelty and value dimensions appear to be mostly independent (i.e., uncorrelated). If true, this finding would have important implications for the assessment of product creativity. In particular, it would imply that product creativity depends on a two-dimensional space organized around novelty and value. Consequently, using only one concept to distinguish between two products on the basis of their creativity (i.e., saying that one product is more creative than another product) would be unwise. Instead, as two factors related to product creativity seem to be distinct from each other, it would be more judicious to make comparisons on these two dimensions (i.e., saying than one product is more or less novel and valuable than another product). Therefore, these results run counter to the way that researchers and the general populations use the term creative.

Other empirical arguments based on regression analyses appear to lend credence to a novelty-based creativity. For example, Acar, Burnett, and Cabra (2017) showed that the novelty criterion explains a substantial part of the variance in creativity judgments, whereas the value criterion only accounts for a small part of this variance. These results also echo those of Diedriech, Benedek, Jauk, and Neubauer (2015), who showed that the novelty effect explains approximately four times more variance than the value effect in creativity judgments. Similarly, Caroff and Besançon (2008) conducted an experimental study and observed that product novelty explained approximately ten times more variance in creativity judgements than value $\left(\eta^{2} p=0.80\right.$ for Novelty vs. 0.06 for value)

Moreover, based this time on correlational analyses between creativity judgements and novelty criteria, Kudrowitz and Wallace (2013) deemed that the high correlation they found 
between the two $(r=.89)$ tended to make separate evaluations of novelty and creativity redundant. Other studies have reached similar conclusions regarding the potential sufficiency of novelty when productions are evaluated by judges (Christiaans, 2002; Chulvi, Mulet, Chakrabarti, López-Mesa, \& González-Cruz, 2012; Runco \& Charles, 1993). Although value is difficult to account for in creativity judgements, this criterion is nonetheless fundamental, and previous results should lead us to consider this attribute separately. Kudrowitz and Wallace (2013, p. 133) expressed this idea very well: "Not all things that are creative are practical or useful (e.g., Dippin' Dots, Disneyworld, and Phillip Stark's Juicy Salif Juicer). These are all very successful and creative products that have no practical use aside from fun and enjoyment". While correlational and regression studies seem difficult to refute on the methodological side $^{4}$, the results suggesting that the novelty and value of creative products are independent are questionable on methodological grounds. In the majority of the above-mentioned studies that applied a psychometric approach to product creativity, the independence of the two dimensions may have stemmed from statistical choices (e.g., the choice to force the dimensions to be independent by performing orthogonal axis rotations such as varimax rotations). In fact, $73.7 \%$ of the studies we identified used orthogonal rotation, while only $26.3 \%$ allowed their dimensions to be correlated using a nonorthogonal rotation method (e.g., oblimin rotation). Moreover, even in these studies that allowed the novelty and value of creative products to be correlated, correlation matrices between factors were almost never reported, making it difficult to conduct an in-depth analysis of a potential relationship between the two dimensions.

Thus, although assumptions are sometimes made about the relationships between the main dimensions underlying the creativity of a product, they are rarely explained and quantified in psychometric studies of creative products. Although they did not adopt a psychometric

\footnotetext{
${ }^{4}$ Other arguments can be made, similar to those cited above for psychometric studies, such as the number and expertise of judges.
} 
approach, as their aim was not to test hypotheses related to the main criteria of product creativity, other studies of creative products (Diedriech, Benedek, Jauk, \& Neubauer, 2015; Kudrowitz \& Wallace, 2013; Mulet, Royo, Chulvi, \& Galán, 2017) have reported correlations between some criteria assumed to load on creativity factors. For instance, in their search for the ingredients of creativity, Diedrich et al. (2015) found a significant negative relationship between usefulness and novelty $(r=-.55)$. Moreover, in a study seeking to analyze the relationship between degree of creativity (assumed to be related to novelty and usefulness) and quality (assumed to be related to effectiveness and feasibility) of productions, Mulet et al. (2017) found that effectiveness is closely related to usefulness $(r=.84)$, and the more novel a production is considered to be, the more it loses its feasibility $(r=-.86)$. These results tend to replicate those of Dean et al. (2006) who, in their CFAs of products resulting from two ideation tasks, found a negative relationship between the novelty and feasibility dimensions they had identified (Task 1: $r=-.79$; Task 2: $r=-.78$ ). Kudrowitz and Wallace (2013) found that feasibility (labelled product-worthiness in their study) and utility were very strongly correlated $(r=.92)$. Taken together, these results suggest that the main dimensions of product creativity are related, and that this relation could be negative (i.e., the more novel a product, the less useful, valuable and feasible it is $)^{5}$.

In the light of these results, we hypothesize that the general axis on which objects differ from each other derives from the antagonism observed between novelty, value, and feasibility. As such, the new concept we introduce here differs from the axis of creativity assumed by the standard definition of creativity (Runco \& Jaeger, 2012), where the most creative objects (i.e., objects with both value and novelty) are at the top, and the least creative at the bottom. On this

\footnotetext{
${ }^{5}$ Nevertheless, it is very important to note that face of the wide variety of possible creative problems, some studies were also able to report moderate and strong significant positive correlations between the criteria for assessing idea quality and novelty (Ford \& Sullivan, 2010; Mumford, Baughman, Maher, Costanza \& Supinski, 1997; Reiter-Palmon, Mumford, O’Connor Boes \& Runco, 1997; Watts, Steele, Medeiros, \& Mumford, 2019). These inconsistencies motivate a study of these relationships.
} 
new axis, the objects at the top are the newest, least valuable, and most unfeasible, while those at the bottom are the most ordinary, most valuable, and most feasible. Therefore, the most creative ideas (in the meaning of the Standard definition of creativity) are those closest to the midpoint of this new axis, or slightly above this midpoint if we lend more weight to novelty than to value and feasibility when defining the creativity of an object.

\section{The Present Study}

The main goal of the present study was to test the existence of relationships between the three main components of creative objects (i.e., novelty, value, and feasibility), and that of a hypothetical second-order general factor revealed by these relationships. To our knowledge, no study had previously attempted to address this issue. As mentioned above, not only did several previous psychometric analyses of object creativity fail to consider feasibility as they examined the creativity of already existing objects, but they also tended to force the independence between novelty and value through debatable statistical choices. Based on the definition of an object's novelty, value and feasibility, and the accumulation of evidence in the literature for a

possible (1) negative relationship between novelty and feasibility, (2) positive relationship between value and feasibility, and (3) distinctiveness between these three dimensions, we tested three hypotheses.

First, we hypothesized that the novelty, feasibility, and value factors are correlated and lead to a second-order general factor of disruptiveness (H1). We called this general factor disruptiveness to underline a subtle difference between the axis formed by the three dimensions and the axis of creativity defined in the literature, where the most creative ideas are at the top and the least creative at the bottom. Instead, it is better to consider this axis as representing degrees of balance between the different dimensions. The most balanced ideas in terms of 
novelty, feasibility and value therefore lie at the midpoint of the axis, while the ideas at the top of the axis have a high level of novelty and low levels of value and feasibility, and vice versa.

The three-factor structure discussed here was compared with other competing structures to ensure that it fits the data. Three competing structures were chosen because of their theoretical and empirical underpinnings. Firstly, considering the previous factorial studies (Amabile, 1979; Besemer \& O'Quin, 1986; Christiaans, 2002; Dean et al., 2006; Demirkan \& Afacan, 2012; Form, Aue, \& Kaernbach, 2019; Horn \& Salvendy, 2006b, 2009; O'Quin \& Besemer, 1989; White \& Smith 2001) that have suggested the independence of the creativity criteria, an independent three-factor structure was tested. Secondly, in line with the arguments that value and feasibility could represent the same variable (Rietzschel, Nijstad \& Stoebe, 2010), a correlated two-factor model as well as an independent two-factor model were also included in the analysis.

That said, the literature tends to show that the concept of creativity is highly positively correlated with novelty and highly negatively correlated with feasibility, so for judgments of creativity, we hypothesized that novelty is positively related to the creativity of ideas $(\mathrm{H} 2 \mathrm{a})$, and feasibility and value are negatively related to the creativity of ideas (H2b). Furthermore, as creativity is a slightly different concept from disruptiveness, according to the standard definition (Runco \& Jaeger, 2012), whereby a creative production must be both novel and valuable, we hypothesized that the general factor of disruptiveness predicts the creativity of an idea in a nonlinear, quadratic manner (H3). Accordingly, as the level of disruptiveness increases, so should the perceived creativity of an idea, but beyond a certain level of disruptiveness, that perceived creativity should weaken.

\section{Method}




\section{Participants and Design}

In order to collect a large number of ideas for evaluation, we recruited a sample of 126 participants $(86.51 \%$ female) aged $17-33$ years $(M=19.52, \mathrm{SD}=1.98)$. The participants were psychology students from the University of Aix-Marseille, and took part in this study in exchange for a course credit. They were asked to produce solutions to the following problem: How to improve the wellbeing of individuals working in a company. The brainstorming activity was divided into two phases of 20 minutes each. In the first phase, participants were instructed to focus primarily on producing as many ideas as possible. During the second phase participants were asked to focus not on the quantity but on the quality of the ideas they produced (instructions are available

at https://osf.io/4fq3a/?view only=92328dab6c1b4b398befba38aeb43f8b). The first phase corresponds to the rules of brainstorming originally stated by Osborn (1957). This phase, which focuses on the quantity of ideas produced, echoes the divergent thinking aimed at expanding the space for proposed solutions without constraints. Nevertheless, this phase, as fundamental as it is to creativity, cannot be a final goal. Some authors (Cropley, 2006; Simonton, 2015) propose extending this phase by adding a convergence phase during which critical reflection is carried out on the ideas produced and/or to be produced. It is through the combination of these two important and opposing phases that the creativity of ideas can potentially be fully expressed. (Paulus, Kohn, \& Arditti, 2011).

We also recruited four experts (two women and two men) in the field of ergonomics/human factors (specialized in the field of work, organizations and health) to judge the ideas produced by the participants. They were aged $26-30$ years $(M=27.5, S D=1.91)$. Two of these judges evaluated all the ideas $(\mathrm{N}=662)$ produced by the participants, on the basis of a questionnaire with 13 items. The other two assessed 57.94\% $(n=383)$ of these ideas. The evaluators were paid either 115 or 200 euros for their work. 


\section{Material}

The questionnaire was constructed on the basis of four existing scales (Creative Product Semantic Scale; Besemer \& O'Quin, 1986, 1987, 1999; O'Quin \& Besemer, 1989; Product Creativity Measurement Instrument; Horn \& Salvendy, 2006a, 2006b, 2009; Creative Solution Diagnosis Scale; Cropley \& Kaufman, 2012; and the scale developed by Dean et al., 2006). Given the large number of ideas we collected from the participants, the main objective in constructing this questionnaire was to have as few items as possible (i.e., conventionally, 3-4 items are needed per dimension to perform robust CFA). In order to include the most relevant items for the three assumed dimensions of creativity, we selected the items with the highest saturations on the novelty and value dimensions. For the novelty dimension, we selected the items new, unusual, surprising, and original from the Creative Product Semantic Scale, the Product Creativity Measurement Instrument, and Dean et al. (2006)'s scale. For the value dimension, we selected the items relevance, effective, useful, and appropriate from the Creative Product Semantic Scale, the Creative Solution Diagnosis Scale, and Dean et al. (2006)'s scale. For the feasibility dimension, as there were very few items in the literature, we based ourselves on Dean et al. (2006), and therefore chose three synonyms of the term feasible (i.e., conceivable, possible, and acceptable). Furthermore, in order to allow for more detailed analyses, we added a 13th item to this scale, namely creative. This item would enable us to observe the extent to which the experts' creativity judgements were predicted by each dimension. The 13 adjectives were rated all at once for a given idea. They were also randomly presented for each idea, to avoid a list effect, and judges were asked to respond on a visual analog scale ranging from Not at all to Totally (unknown to participants, this was actually a 100-point scale).

\section{Data Analytic Strategy}

In this study the unit of analysis was the individual ideas and the data analytic strategy came in three phases. First, descriptive statistics were computed for all of the variables of 
interest. More specifically, means, standard deviations, and skewness coefficients were calculated for each of the 13 items. To provide initial information about the pattern of their covariations, correlations between these items were also computed. A matrix of the correlations between 13 variables can be hard to interpret, as it necessarily contains 78 numerical values. Therefore, the pattern of covariations was also displayed in graphic form, using a sparse Gaussian model (Epskamp, Borsboom, \& Fried, 2018). As well as serving inferential purposes, such graphical models can also be used at a descriptive level, as they consist of parsimonious, easily interpretable, networks made up solely of the strongest correlations between the variables examined (Epskamp et al., 2018) ${ }^{6}$.

Second, to test the hypotheses regarding the main dimensions underlying the creativity of ideas, a series of CFA models with maximum likelihood estimation was computed, and compared with each other. These models were estimated on the 12 items selected to assess novelty, feasibility, and value. The main model hypothesized in the present study (i.e., the three correlated factors model) was compared, as mentioned above, to three reasonable alternatives (i.e., a three uncorrelated factors model, a two correlated factors model, and two uncorrelated factors model). To compare these models on their ability to account for the actual covariation structure between the items, four common fit indices were used: comparative fit index (CFI), Tucker-Lewis index (TLI), root mean square error of approximation (RMSEA), and standardized root mean square residual (SRMR). By convention, a value above 0.90 for the CFI or TLI, and a value below 0.08 for the RMSEA or SRMR, indicate an acceptable fit (Brown \& Cudeck, 1993).

\footnotetext{
${ }^{6}$ In the psychology literature, the sparsity of sparse Gaussian graphical models (i.e., their capacity to solely represent the strongest correlations between the variables examined) is generally achieved by (1) focusing on partial correlations, and (2) using the least absolute shrinkage and selection operator (LASSO) regularization parameter. Minimizing the extended Bayesian information criterion represents the tuning parameter used by LASSO to determine the degree to which regularization is applied (Epskamp et al., 2018).
} 


\section{Table 1}

Confirmatory Factor Analysis Models Compared

\section{Label}

Structure

F1 $=\mathrm{New}+\mathrm{Ori}+\mathrm{Sur}+\mathrm{Unu}$

1. Two uncorrelated factors model

$\mathrm{F} 2=\mathrm{Pos}+\mathrm{Con}+\mathrm{Unf}+\mathrm{Acc}+\mathrm{Eff}+\mathrm{Rel}+\mathrm{Use}+\mathrm{App}$

2. Two correlated factors model

$$
\begin{aligned}
& \mathrm{F} 1=\mathrm{New}+\mathrm{Ori}+\mathrm{Sur}+\mathrm{Unu} \\
& \mathrm{F} 2=\mathrm{Pos}+\mathrm{Con}+\mathrm{Unf}+\mathrm{Acc}+\mathrm{Eff}+\mathrm{Rel}+\mathrm{Use}+\mathrm{App} \\
& \mathrm{GF}=\mathrm{F} 1+\mathrm{F} 2
\end{aligned}
$$

$$
\begin{aligned}
& \mathrm{F} 1=\mathrm{New}+\text { Ori }+ \text { Sur }+ \text { Unu } \\
& \text { F2 }=\text { Pos }+ \text { Con }+ \text { Unf }+ \text { Acc } \\
& \text { F3 }=\text { Eff }+ \text { Rel }+ \text { Use }+ \text { App }
\end{aligned}
$$

$$
\begin{aligned}
& \text { F1 }=\text { New }+ \text { Ori }+ \text { Sur }+ \text { Unu } \\
& \text { F2 }=\text { Pos }+ \text { Con }+ \text { Unf }+ \text { Acc } \\
& \text { F3 }=\text { Eff }+ \text { Rel }+ \text { Use }+ \text { App } \\
& \text { GF }=\text { F1 }+ \text { F2 }+ \text { F3 }
\end{aligned}
$$

Note. $N=$ novelty; $F=$ feasibility; $V=$ value $; F V=$ factor combining feasibility and value; $N F$ $=$ factor combining novelty and feasibility; $F \#=$ factor number; $G=$ general; = = saturating; $+=$ and; Ori = original; Sur = surprising; Unu = unusual; Pos = possible; Con = conceivable; Unf = unfeasible; Acc = acceptable; Eff = effective; Rel = relevant; Use = useless; App = appropriate. The model in bold is the model hypothesized to have the best fit in the present study.

Third, to test the hypotheses regarding the dependence of creativity judgments on the main dimensions underlying the creativity of a given idea, scores for all the factors present in the CFA model with the best fit were computed. These factor scores enabled the estimation of latent variables such as novelty or feasibility. Then, as in previous research (e.g., Acar, Burnett, \& Cabra, 2017; Diedrich, Benedek, Jauk, \& Neubauer, 2015), ordinary least squares regressions were ran, where the outcome variable was the degree to which an idea was judged to be creative. Like the predictor variables, they contained the scores of the factors identified by the CFA 
model with the best fit. These analyses constituted a straightforward test of the degree to which a judgment of creativity depends on the particular ingredients of the idea (i.e., its novelty, feasibility, and value).

All analyses were performed using R (R Core Team, 2019). The bootnet (Epskamp et al., 2018) and qgraph (Epskamp, Cramer, Waldorp, Schmittmann, \& Borsboom, 2012) R packages were used to estimate and represent the above-mentioned sparse Gaussian graphical model, while the lavaan (Rosseel, 2012), psych (Revelle, 2019) and semPlot (Epskamp, 2019) $\mathrm{R}$ packages were used to estimate and represent the above-mentioned CFA models. The dataset on which the analyses were based and the $\mathrm{R}$ code used are contained in an open-access file available at: https://osf.io/4fq3a/?view_only=92328dab6c1b4b398befba38aeb43f8b

\section{Results}

\section{Interrater Reliability}

Interrater reliability was assessed using a two-way random, consistency, averagemeasures intraclass correlation coefficient (ICC) (Cseh \& Jeffries, 2019; McGraw \& Wong, 1996) to assess the degree of consistency in the judges' ratings of the 13 items for each idea/solution. As all four judges evaluated $57.94 \%$ of the proposed solutions $(n=383)$ and two of them evaluated all the solutions $(N=662)$, two separate analyses were conducted.

The ICC for the two judges who assessed all of the ideas are displayed in Table 2. They ranged from $\operatorname{ICC}(\mathrm{C}, 2)=.513$ to $\operatorname{ICC}(\mathrm{C}, 2)=.74\left(M_{\mathrm{ICC}}=.65, S D_{\mathrm{ICC}}=.06\right)$, so from "moderate" to "high" (Cicchetti, 1994; Koo \& Li, 2016), indicating that the judges had at least a moderate degree of agreement and suggesting that all 13 items were rated fairly similarly across judges.

The ICC for the four judges (Table 2) who assessed 57.94\% $(n=383)$ of the ideas ranged from ICC $(\mathrm{C}, 4)=.722$ to $\mathrm{ICC}(\mathrm{C}, 4)=.832\left(M_{\mathrm{ICC}}=.78, S D_{\mathrm{ICC}}=.03\right)$, so from high to almost 
perfect (Cicchetti, 1994; Koo \& Li, 2016), indicating a high degree of interrater agreement and suggesting that all 13 items were rated similarly across judges.

\section{Table 2}

Descriptive statistics and intercorrelations between the variables of interest of the present study

\begin{tabular}{|c|c|c|c|c|c|c|c|c|c|c|c|c|c|}
\hline & 1 & 2 & 3 & 4 & 5 & 6 & 7 & 8 & 9 & 10 & 11 & 12 & 13 \\
\hline$M$ & 54.79 & 55.07 & 58.79 & 61.69 & 74.87 & 77.75 & 28.69 & 80.78 & 72.62 & 68.19 & 27.29 & 69.42 & 52.52 \\
\hline$S D$ & 30.72 & 29.83 & 30.94 & 30.36 & 24.82 & 22.99 & 26.28 & 23.43 & 24.02 & 28.05 & 26.02 & 29.04 & 27.63 \\
\hline$I C C_{2}$ & 0.70 & 0.68 & 0.67 & 0.68 & 0.62 & 0.51 & 0.65 & 0.60 & 0.61 & 0.68 & 0.64 & 0.74 & 0.64 \\
\hline$I C C_{4}$ & 0.80 & 0.78 & 0.80 & 0.81 & 0.79 & 0.72 & 0.78 & 0.79 & 0.76 & 0.81 & 0.79 & 0.83 & 0.72 \\
\hline \multicolumn{14}{|l|}{ 1. New } \\
\hline 2. Ori & 0.94 & & & & & & & & & & & & \\
\hline 3. Sur & 0.92 & 0.90 & & & & & & & & & & & \\
\hline 4. Unu & 0.91 & 0.89 & 0.93 & & & & & & & & & & \\
\hline 5. Pos & -0.62 & -0.57 & -0.63 & -0.60 & & & & & & & & & \\
\hline 6. Con & -0.57 & -0.52 & -0.58 & -0.56 & 0.80 & & & & & & & & \\
\hline 7. $U n f$ & 0.64 & 0.60 & 0.64 & 0.62 & -0.90 & -0.76 & & & & & & & \\
\hline 8. $A c c$ & -0.46 & -0.42 & -0.50 & -0.47 & 0.63 & 0.79 & -0.59 & & & & & & \\
\hline 9. Eff & -0.36 & -0.34 & -0.40 & -0.36 & 0.49 & 0.66 & -0.44 & 0.77 & & & & & \\
\hline 10. Rel & -0.52 & -0.50 & -0.58 & -0.54 & 0.63 & 0.73 & -0.59 & 0.80 & 0.86 & & & & \\
\hline 11. Use & 0.48 & 0.45 & 0.52 & 0.50 & -0.59 & -0.71 & 0.56 & -0.79 & -0.85 & -0.91 & & & \\
\hline 12. App & -0.55 & -0.54 & -0.59 & -0.56 & 0.65 & 0.77 & -0.62 & 0.84 & 0.83 & 0.93 & -0.87 & & \\
\hline 13. Cre & 0.87 & 0.91 & 0.83 & 0.83 & -0.47 & -0.38 & 0.51 & -0.28 & -0.23 & -0.39 & 0.35 & -0.42 & \\
\hline
\end{tabular}

Note. Ori $=$ original; Sur $=$ surprising; Unu $=$ unusual; Pos $=$ possible $;$ Con $=$ conceivable; Unf = unfeasible; Acc $=$ acceptable $;$ Eff $=$ effective $;$ Rel $=$ relevant $;$ Use $=$ useless $;$ App $=$ appropriate; Cre $=$ creative. All correlations were statistically significant at $p<.001$.

\section{Descriptive Statistics}


Descriptive statistics are displayed in Table 2 and Figure 1. Four interesting results emerged at this initial descriptive level. First, all the variables were correlated with each other in a non-negligible manner. When the focus was restricted to the 12 items designed to assess different features of novelty, feasibility, and value, the minimum correlation that emerged was moderate in size (i.e., $r=-.34$ between originality and effectiveness). When the item that directly assessed creativity was also considered, the minimum correlation that emerged was nonnegligible in size (i.e., $r=-.23$ between creativity and effectiveness). Thus, as expected, a general factor seemed to subtend all the variables.

Second, the above-mentioned general factor appeared to distinguish the most novel / constraint-breaking (unfeasible) / ineffective ideas from the most ordinary / constraintrespecting (feasible) / effective ones. For instance, idea scores on the new item were negatively correlated with their scores on the possible $(r=-.62)$ and effective $(r=-.36)$ items. Thus, as expected, the general factor underlying all the variables in the present study seemed to reflect an axis of disruptiveness, where the most novel ideas tended to be the least constraint-respecting (feasible) and valuable ones.

Third, albeit correlated, three subgroups of variables emerged. As expected, these three subgroups reflected novelty, feasibility, and value. More specifically, items designed to assess the same ingredient of creativity (e.g., novelty) tended to be more strongly correlated than items designed to assess different ingredients (e.g., novelty and feasibility). For instance, the correlation between the items new and original was .94 , whereas the correlation between the items new and possible was -.62, and the correlation between the items new and effective was just -.36. Relationships between the variables examined in the three subgroups is clearly observable in Figure 1, where the distance between variables was determined by the Fruchterman-Reingold algorithm, which place strongly correlated variables close to each other (see Epskamp et al., 2012). 
Fourth, as expected, the degree to which an idea was judged to be creative seemed to be correlated more closely with its perceived novelty than with its perceived feasibility and value. For instance, idea scores on the item directly assessing creativity were more closely correlated with scores on the item new $(r=.87)$ than with scores on the items possible $(r=-.47)$ and effective $(r=-.23)$. Moreover, in Figure 1, this item was placed by the Fruchterman-Reingold algorithm within the subgroup of items designed to assess novelty, suggesting strong correlations with those items.

Taken together, these observations were consistent with what was expected. However, in order to test the hypotheses on the structure of the ingredients of idea creativity and their relationship to the perceived creativity of these ideas, inferential statistics were required.

Importantly, three items were negatively skewed and one item was positively skewed, nevertheless, we chose not to transform these variables (e.g., log transform them), because the residuals of all the models reported below were normally distributed. 


\section{Figure 1}

Network of the partial correlations between the variables of interest.

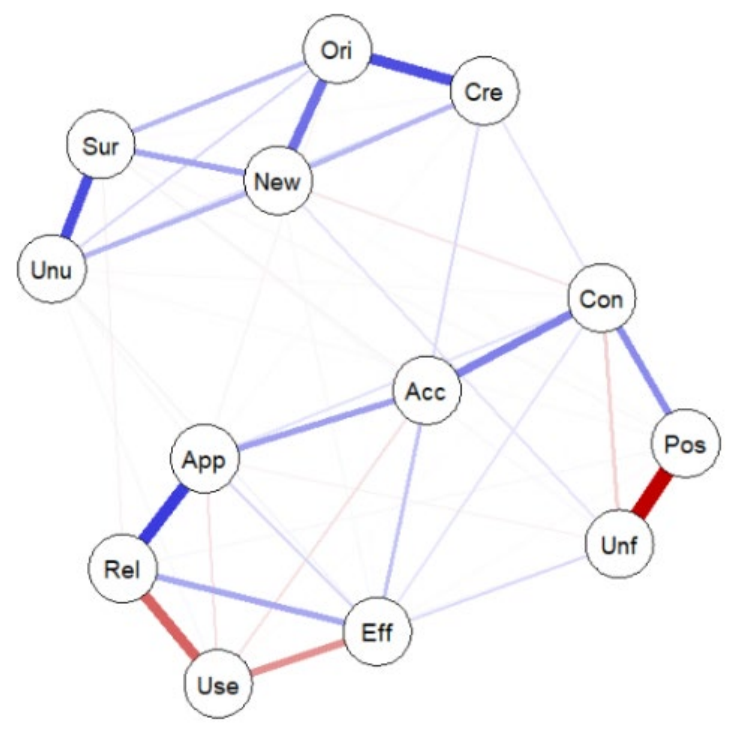

Note. Ori = original; Sur = surprising; Unu = unusual; Pos = possible; Con = conceivable; Unf = unfeasible; Acc = acceptable; Eff = effective; Rel = relevant; Use = useless; $A p p=$ appropriate; $C r e=$ creative. Blue lines represent positive correlations, and red lines negative correlations. The width of the lines is proportional to the strength of the correlations represented. The proximity of two variables also depends on the strength of their correlation.

\section{Structure of Idea Creativity: Confirmatory Factor Analysis Model Comparison}

Aa CFA model with three first-order factors and one second-order general factor, as well as three possible alternative models (see Table 1), were computed.

Results are displayed in Table 3 and Figure 2. The three alternative models showed a poor fit on all the common fit indices used in the present study. By contrast, the hypothesized model showed an acceptable fit on three of the four fit indices $(\mathrm{CFI}=0.95, \mathrm{TLI}=0.93, \mathrm{RMSEA}$ $=0.14, \mathrm{SRMR}=0.06)$. To determine whether this better fit was statistically significant, a $\chi^{2}$ difference test for CFA model comparison was computed. It was computed for the difference between the hypothesized model and the alternative model with the best fit (i.e., three uncorrelated factors model). As expected, the difference was statistically significant $\left(\chi^{2}\right.$ difference $=785, p<.001$ ). Thus, the model stating that novelty, feasibility, and value are three 
distinct but correlated factors explained the structure of the covariations between the items probing creativity in an acceptable manner, and significantly better than realistic alternative models.

Moreover, Figure 2 corroborates the idea that the correlations between novelty, feasibility, and value led to the emergence of a second-order factor of disruptiveness. More specifically, this general factor positively loaded on novelty, and negatively loaded on feasibility and value. In other words, as hypothesized and already observed in the descriptive statistics, this general factor distinguished the most novel / constraint-breaking (unfeasible) /ineffective ideas from the most ordinary / constraint-respecting (feasible) / effective ones. The most novel ideas actually tended to be the least constraint-respecting (feasible) and valuable ones, and the general factor identified precisely captured these between-idea differences. 
Table 3

Fit Indices for the Seven Confirmatory Factor Analysis Models Tested

\begin{tabular}{lcccccccc}
\hline \multicolumn{1}{c}{ CFA model } & $\chi^{2}$ & df & CFI & TLI & RMSEA & SRMR & AIC & BIC \\
\hline $\begin{array}{l}\text { 1. Two uncorrelated factors } \\
\text { (N/FV) model }\end{array}$ & 1947 & 54 & 0.84 & 0.80 & 0.23 & 0.35 & 65414 & 65522 \\
$\begin{array}{l}\text { 2. Two correlated factors } \\
\text { (N/FV) model }\end{array}$ & 1676 & 52 & 0.86 & 0.84 & 0.22 & 0.11 & 65147 & 65264 \\
$\begin{array}{l}\text { 3. Three uncorrelated factors } \\
\text { model }\end{array}$ & 1448 & 53 & 0.88 & 0.85 & 0.20 & 0.43 & 64916 & 65029 \\
$\begin{array}{l}\text { 4. Three correlated factors } \\
\text { model }\end{array}$ & 663 & 51 & 0.95 & 0.93 & 0.14 & 0.06 & 64136 & 64257 \\
\hline
\end{tabular}

Note. $d f=$ degrees of freedom, $C F I=$ comparative fit index; $T L I=$ Tucker-Lewis index; RMSEA = root mean square error of approximation; $S R M R$ = standardized root mean square residual; AIC $=$ Akaike information criterion; $B I C=$ Bayesian information criterion.

\section{Figure 2}

Graphical representation of the confirmatory factor analysis model with the best fit.

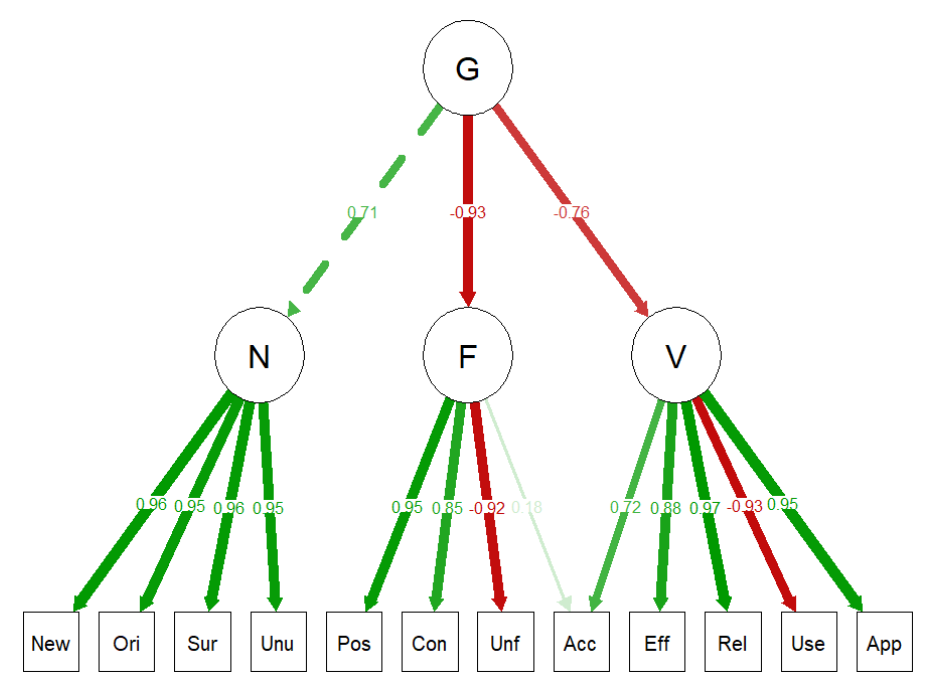

Note. $G=$ general factor; $N=$ novelty; $F=$ feasibility; $V=$ value; Ori = original; Sur = surprising; Unu = unusual; Pos = possible; Con = conceivable; Unf = unfeasible; Acc = acceptable; Eff = effective; Rel = relevant; Use = useless; App = appropriate.

Predictiveness of Main Ingredients of Idea Creativity: Ordinary Least Squares Regressions 
Five ordinary least squares regressions were ran. All these models contained the to which ideas were judged to be creative as the outcome variable. The predictor variables were the latent factors identified in the above-described CFA model, operationalized by their respective factor scores. More specifically, the first regression contained novelty as the predictor variable, the second regression feasibility, the third regression value, the fourth regression novelty, feasibility, and value (considered simultaneously to identify possible changes in the effects when partial rather than simple effects were examined), and the fifth regression the general factor of disruptiveness (linear and quadratic).

Results are displayed in Table 4. As hypothesized, when novelty, feasibility, and value were considered separately, novelty was positively related to idea creativity $(\beta=0.89, p<.001)$, whereas feasibility $(\beta=-0.48, p<.001)$ and value $(\beta=-0.34, p<.001)$ were negatively related to idea creativity.

\section{Table 4}

Standardized Regression Coefficients and 95\% Confidence Intervals of the Five Regression Models Estimated to Predict the Perceived Creativity of the Ideas Examined

\begin{tabular}{|c|c|c|c|c|c|}
\hline Predictor & Model 1 & Model 2 & Model 3 & Model 4 & Model 5 \\
\hline Novelty & $\begin{array}{c}0.89 * \\
{[0.85} \\
0.92]\end{array}$ & & & $\begin{array}{c}1.01 * \\
{[0.96,1.05]}\end{array}$ & \\
\hline Feasibility & & $\begin{array}{c}-0.48^{*} \\
{[-0.55,-0.42]}\end{array}$ & & $\begin{array}{c}0.10^{*} \\
{[0.05,0.15]}\end{array}$ & \\
\hline Value & & & $\begin{array}{c}-0.34^{*} \\
{[-0.41,-0.26]}\end{array}$ & $\begin{array}{c}0.11^{*} \\
{[0.07,0.16]}\end{array}$ & \\
\hline $\begin{array}{l}\text { Disruptiveness } \\
\text { linear }\end{array}$ & & & & & $\begin{array}{c}0.64^{*} \\
{[0.59,0.69]}\end{array}$ \\
\hline $\begin{array}{l}\text { Disruptiveness } \\
\text { nonlinear }\end{array}$ & & & & & $\begin{array}{c}-0.36^{*} \\
{[-0.41,-0.31]}\end{array}$ \\
\hline $\mathrm{R}^{2}$ & 79.14 & 23.34 & 11.36 & 81.28 & 53.90 \\
\hline
\end{tabular}


Note. $R^{2}=$ percentage of explained variance. The first numerical value in each case is the standardized regression coefficient ( $\beta$ ), followed by 95\% confidence intervals into brackets.

Some relationships changed when novelty, feasibility, and value were simultaneously entered as predictor variables in the regression, which allowed the examination of each of these three variables while statistically neutralizing the two others. In particular, when novelty and value were neutralized (i.e., when ideas with identical levels of novelty and value were considered), feasibility was positively related to creativity $(\beta=0.10, p<.001)$. Likewise, when novelty and feasibility were neutralized (i.e., when ideas with identical levels of novelty and feasibility were considered), value was positively related to creativity $(\beta=0.11, p<.001)$. However, these relationships remained much weaker (i.e., approximately 10 times weaker) than the positive relationship between novelty and idea creativity $(\beta=1.01, p<.001)$. Observing a $\beta$ coefficient above 1 for this partial effect of novelty on idea's perceived creativity was not surprising, considering (1) the strong size of the simple effect of novelty on idea's perceived creativity $\beta=0.89$ ), and (2) that $\beta s$ above 1 can emerge when strongly-correlated predictors are simultaneously entered in a regression model. Nevertheless, to ensure that the correlations between the three predictors examined in this regression analysis did not raise serious multicollinearity issues, variance inflation factors were computed. Variance inflation factors are known to indicate multicollinearity issues when they exceed 5 (James, Witten, Hastie, \& Tibshirani, 2014). Results suggested that multicollinearity issues were negligible in this model, as no variance inflation factor exceeded the threshold (variance inflation factor $=1.76,2.21$, and 1.74 for novelty, feasibility, and value, respectively).

As the partial contributions of novelty, feasibility, and value were all positive, and as novelty contributed more strongly to idea creativity than either feasibility or value, we surmised that the disruptiveness axis, which captured the fact that the most novel ideas tended to be the least feasible and valuable ones, had a nonlinear relationship with idea creativity. This was 
confirmed by observations, as in the fifth regression ran, both linear disruptiveness $(\beta=0.64, p$ $<.001)$ and quadratic disruptiveness $(\beta=-0.35, p<.001)$ were significantly related to creativity. Predictions were derived from the coefficients estimated by this fifth regression to graphically represent the nonlinear relationship between disruptiveness and creativity (see Figure 3). As hypothesized, as the level of disruptiveness increased, so the perceived creativity of an idea increased, but beyond a certain level of disruptiveness (i.e., around one standard deviation above the mean), that perceived creativity weakened slightly.

\section{Figure 3}

Graphical representation of the quadratic effect of the general factor of idea disruptiveness on the perceived creativity of these ideas.

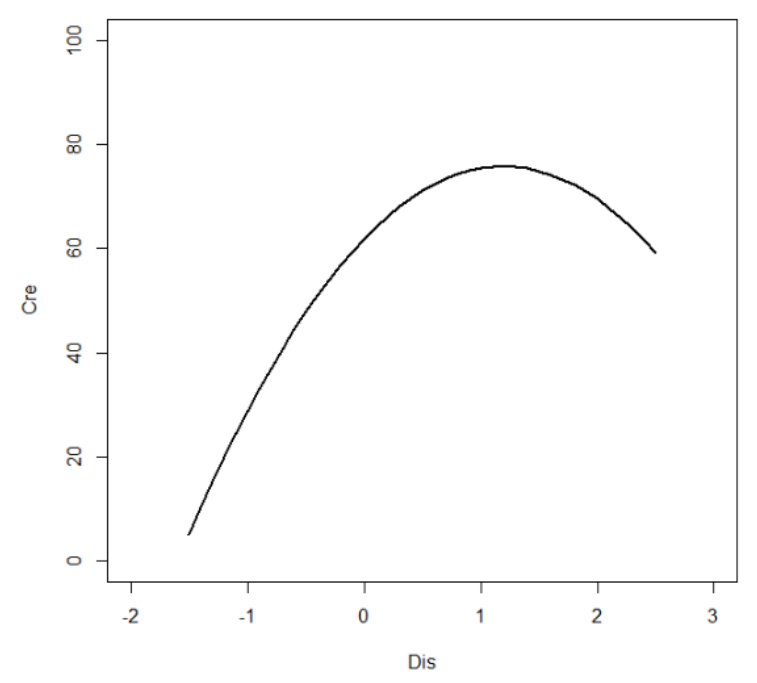

Note. Dis = disruptiveness; Cre = creativity. The latent variable of disruptiveness is standardized $(M=0, S D=1)$, whereas the observed variable of creativity is on the raw scale. The curve goes from -1.5 to 2.5 on the $x$-axis because the minimum disruptiveness value was 1.48 and the maximum value was 2.56 .

\section{Discussion}

Although the results obtained in the present study can be considered with caution due to the limitations that we will detail below; the main result of the present study was that creative 
ideas appear to differ from each other on a general disruptiveness axis revealed by the relationships between the objects' novelty, value, and feasibility. The most novel ideas tend to be judged as the least valuable and feasible ones. In other words, the more novelty an object acquires, the more value and feasibility it loses. This result appears to run counter to the results of previous psychometric attempts to identify the main dimensions on which creative objects differ from each other. There are two main explanations for this inconsistency. First, in the majority of these previous attempts, statistical choices (i.e., performing factor analyses with varimax rotation) forced the identified dimensions (e.g., novelty, value) to be independent, even though they might actually be correlated (Amabile, 1979; Besemer \& O'Quin, 1986; Christiaans, 2002; Demirkan \& Afacan, 2012; Form et al., 2019; Horn \& Salvendy, 2006b, 2009; O'Quin \& Besemer, 1989; White \& Smith, 2001). Second, the majority of these previous attempts focused on creative objects that already existed, and thus overlooked feasibility (Amabile, 1979; Besemer, 1998; Besemer \& O’Quin, 1986, 1999; Christiaans, 2002; Demirkan \& Afacan, 2012; Form et al., 2019; Horn \& Salvendy, 2006b, 2009; O’Quin \& Besemer, 1989; White \& Smith, 2001). Nevertheless, it is important to note that although the aim of these studies was not to psychometrically derive the main dimensions of object creativity, some previous studies had already documented a strong negative correlation between novelty and value or feasibility, and a strong positive correlation between value and feasibility (Diedrich et al., 2015; Kudrowitz \& Wallace, 2013; Mulet et al., 2017).

The disruptivness axis seems to have interesting theoretical and practical implications that need to be put into perspective. From a practical point of view, this axis seems to be an interesting tool for making decisions and selecting the most creative ideas. If the goal of creativity is to come up with an idea that is strong in originality, feasibility and value, the operationalization of this axis of disruptiveness makes it possible, through the selection of the 
most balanced ideas, to guide the decision-making process and, through an iteration cycle of idea/solution selection, to control the dynamic development of that idea.

Creativity as a dynamic process seem to echo the dynamic definition of creativity proposed by Corazza (2016), which emphasizes the importance of two criteria: potential originality and potential effectiveness. According to this definition, creativity is first and foremost a dynamic process that requires the creator to go through many inconclusive creations before reaching the expected goal. Most of the creative ideas/solutions that have given rise to innovations have not appeared instantaneously, but are the result of a lengthy process of reflection, in which decision-making is a key element. The disruptiveness axis responds to this need by providing a tool that allows researchers to better understand the relationships between the supposed dimensions of creativity, and by offering the applied world a decision-making tool supported by empirical research arguments. Nevertheless, the above-mentioned ideas should be viewed with caution, considering the present study's limitations.

The first main limitation concerns the type of problem statement. In the present study, the problem was intended to be as ecological as possible and was consequently ill-defined. Therefore, although there were some validity constraints in the problem, there were far more preference constraints, thus allowing participants to produce ideas that could have value but whose feasibility might vary quite considerably. As long as they respected explicit validity constraints (i.e., improving the wellbeing of office workers), participants could afford to produce ideas that, for example, seemed technically or economically unfeasible. Thus, depending on the type of problem and the way it is defined, this dimension can be merged with the value dimension (i.e. problem constraints shifting from preference to validity, and vice versa). Moving from preference constraints to validity constraints tends to reduce the space for possible solutions. If we had defined that in economic terms, the ideas should cost less than 20 euros, the set of possible solutions above this sum of money would have become irrelevant to 
solving the problem. Finally, just as there are conditions where feasibility is merged with value, there are conditions where value seems to be evaluated only dichotomously. In such a framework, off-topic ideas are distinguished from relevant ideas (e.g., the alternate uses tasks commonly used as divergent-thinking task). In such tasks, novelty (statistical originality) is the only characteristic that can be evaluated. Future research should seek to corroborate or refute this structure with other kind of tasks.

The second limitation is related to the domain. The dimensions associated with constraints such as value and feasibility do not necessarily have the same influence, depending on the characteristics of the domain. For example, the subjectivity associated with value in the field of art or with user-centered design may lead to highly variable scores, whereas in fields known to be more objective, such as science or engineering design, value could be relatively stable.

The third limitation that could also have an influence on the factor structure of the creativity of productions could also be the type and/or expertise of judges used (for further detail on issues concerning the consensual assessment technique limitations; see Cseh \& Jefferies, 2019). For example, since the problem we are using in this study involves users (i.e., employee working for company), the ideas obtained could also have been evaluated by the end users put forward by the problem on the value dimension (employees working in the organization). It would also be interesting to use new assessment approaches such as the "Response Theory Judge" as proposed by Myszkowski \& Storme (2019)

The existence of moderators (e.g., type of problem, type of judge, type of instructions, etc.) probably explains the existence of variability from one study to another in the results obtained on the relationships between quality (value, feasibility) and originality. Indeed, some studies have shown that the instructions given to participants can also influence different measures of creativity (Forthmann et al., 2016; Reiter-Palmon \& Arreola, 2015) and thus its 
structure. In other studies, significant positive relationships between novelty and value (or qualitative criteria) of a product (Mumford, Baughman, Maher, Costanza \& Supinski, 1997; Reiter-Palmon, Mumford, O’Connor Boes \& Runco, 1997; Watts, Steele, Medeiros, \& Mumford, 2019) have even been identified. A particularly interesting example is Ford \& Sullivan (2010). These researchers obtained positive correlations (moderate to strong) between their indicators of novelty and value (e.g., correlation between "originality" and "valuable" was: $r=.50)$. However, it is important to note that their studies only focused on an extremely specific case. These researchers only focused on between-judge differences rather than between-idea differences. More specifically they considered the extent to which dozens of judges differed from each other in the way they evaluated one single creative highly creative idea

Future research is needed to identify these moderators. Indeed, to date and to our knowledge, there are no theoretical arguments or empirical studies that have sought to understand the conditions under which novelty and value have positive, neutral or negative relationships. If we think that, in general, the more novel a production is, the less value and/or feasibility it has, there are certainly cases in which these relations disappear in the opposite direction. Again, these cases may give rise to future studies focusing on these fundamental issues.

Another limitation of the present study was that possible biases related to common method variance were not controlled for. The most effective strategy currently available to control for such possible biases is to add marker variables to the protocol (Simmering, Fuller, Richardson, Ocal, \& Atinc, 2015; Williams, Hartman, \& Cavazotte, 2010), a strategy which would have resulted in the addition of items to the 13-item questionnaire used, and therefore in a more important load for the judges. In future research, however, it would be possible to remove some items to the 13-item questionnaire used in this study (e.g., the item loading the 
least on its factor) to enable the addition of other items assessing a marker variable without adding load on the judges. This would be an important research to conduct in order to determine the extent to which correlations between the items used in this study can be increased by methodological characteristics.

A last limitation regarded the values of the fit indices computed. The three-correlated factor model fitted data in an acceptable manner according to the so-called incremental fit indices (i.e., CFI and TLI), whereas it did it differently according to the so-called absolute fit indices (i.e., RMSEA and SRMR). More specifically, RMSEA indicated poor fit whereas SRMR indicated acceptable fit. As the main difference between RMSEA and SRMR is that, as compared with RMSEA, SRMR does not contain any penalty for model complexity, the following hypothesis can be formulated. Our model of interest might be too complex (i.e., too many parameters freed) to reproduce data in a parsimonious manner. Finding a more parsimonious model to account for the actual structure of creative ideas might therefore be an important goal to pursue in future research."

To conclude, one main finding emerged from the present study: objects that are perceived to be the most novel ones also tend to be judged the least valuable and feasible. Creative objects therefore appear to differ from each other on a disruptiveness axis. The more novelty an object acquires, the more it tends to lose value and feasibility. This finding may influence the way we define and assess the creativity of objects, as well as the way we intervene to enhance individuals' ability to produce objects that are both novel and valuable. However, given the many limitations of this study, future psychometric studies should be conducted using different parameters to determine the robustness of this result and its possible boundary conditions. 


\section{References}

Acar, S., Burnett, C., \& Cabra, J. F. (2017). Ingredients of creativity: Originality and more.

Creativity Research Journal, 29(2), 133-144.

https://doi.org/10.1080/10400419.2017.1302776

Amabile, T. M. (1979). Effects of external evaluation on artistic creativity. Journal of Personality and Social Psychology, 37(2), 221-233. https://doi.org/10.1037/0022-3514.37.2.221

Besemer, S. P., \& O’Quin, K. (1986). Analyzing creative products: Refinement and test of a judging instrument. The Journal of Creative Behavior, 20(2), 115-126.

https://doi.org/10.1002/j.2162-6057.1986.tb00426.x

Besemer, S. P., \& O'Quin, K. (1987). Creative product analysis: Testing a model by developing a judging instrument. Frontiers of Creativity Research: Beyond the Basics, 367-389.

Besemer, S. P. (1998). Creative product analysis matrix: Testing the model structure and a comparison among products--Three novel chairs. Creativity Research Journal, 11(4), 333346. https://doi.org/10.1207/s15326934crj1104_ 7

Besemer, S. P., \& O’Quin, K. (1999). Confirming the three-factor creative product analysis matrix model in an American sample. Creativity Research Journal, 12(4), 287-296.

https://doi.org/10.1207/s15326934crj1204_6

Besemer, S. P., \& Treffinger, D. J. (1981). Analysis of creative products: Review and synthesis.

The Journal of Creative Behavior, 15(3), 158-178. https://doi.org/10.1002/j.2162$\underline{6057.1981 . t b 00287 . x}$

Browne, M. W., \& Cudeck, R. (1993). Alternative ways of assessing model fit. In K. A. Bollen \& J. S. Long (Eds.), Testing structural equation models (pp. 136-162). Sage.

Caroff, X., \& Besançon, M. (2008). Variability of creativity judgments. Learning and individual differences, 18(4), 367-371. https://doi.org/10.1016/j.lindif.2008.04.001 
Christiaans, H. H. C. M. (2002). Creativity as a design criterion. Creativity Research Journal, 14(1), 41-54. https://doi.org/10.1207/S15326934CRJ1401_4

Chulvi, V., Mulet, E., Chakrabarti, A., López-Mesa, B., \& González-Cruz, C. (2012). Comparison of the degree of creativity in the design outcomes using different design methods. Journal of Engineering Design, 23(4), 241-269. https://doi.org/10.1080/09544828.2011.624501

Cropley, A. J. (2005) Creativity and problem-solving: Implications for classroom assessment. British Psychological Society

Cropley, D. H., \& Cropley, A. J. (2005). Engineering creativity: A systems concept of functional creativity. In J. C. Kaufman \& J. Baer (Eds.), Creativity across domains: Faces of the muse (pp. 169-185). Lawrence Erlbaum Associates.

Cropley, D. H., \& Cropley, A. J. (2008). Elements of a universal aesthetic of creativity. Psychology of aesthetics, creativity, and the arts, 2(3), 155-161. https://doi.org/10.1037/1931-3896.2.3.155

Cropley, D. H., \& Cropley, A. J. (2010). Recognizing and fostering creativity in technological design education. International Journal of Technology and Design Education, 20(3), 345358. https://doi.org/10.1007/s10798-009-9089-5

Cropley, D. H., \& Kaufman, J. C. (2012). Measuring functional creativity: Non-expert raters and the Creative Solution Diagnosis Scale. The Journal of Creative Behavior, 46(2), 119-137. https://doi.org/10.1002/jocb.9

Cropley, D. H., Kaufman, J. C., \& Cropley, A. J. (2011). Measuring creativity for innovation management. Journal of Technology Management \& Innovation, 6(3), 13-30. https://doi.org/10.4067/S0718-27242011000300002

Cseh, G. M., \& Jeffries, K. K. (2019). A scattered CAT: A critical evaluation of the consensual assessment technique for creativity research. Psychology of Aesthetics, Creativity, and the Arts, 13(2), 159-166.https://doi.org/10.1037/aca0000220 
Csikszentmihályi, M., \& Wolfe, R. (2014). New conceptions and research approaches to creativity: Implications of a systems perspective for creativity in education. In M. Csikszentmihalyi (Ed.), The systems model of creativity (pp. 161-184). Springer Netherlands. https://doi.org/10.1007/978-94-017-9085-7 10

Dean, D. L., Hender, J. M., Rodgers T. L., \& Santanen, E. (2006). Identifying good ideas: Constructs and scales for idea evaluation. Journal of Association for Information Systems, 7(10) 646-699.

Demirkan, H., \& Afacan, Y. (2012). Assessing creativity in design education: Analysis of creativity factors in the first-year design studio. Design Studies, 33(3), 262-278. https://doi.org/10.1016/j.destud.2011.11.005

Diedrich, J., Benedek, M., Jauk, E., \& Neubauer, A. C. (2015). Are creative ideas novel and useful? Psychology of Aesthetics, Creativity, and the Arts, 9(1), 35-40. https://doi.org/10.1037/a0038688

Diehl, M., \& Stroebe, W. (1987). Productivity loss in brainstorming groups: Toward the solution of a riddle. Journal of Personality and Social Psychology, 53(3), 497-509. $\underline{\text { https://doi.org/10.1037/0022-3514.53.3.497 }}$

Di Mascio, R., Kalyuga, S., \& Sweller, J. (2018). The effect of wording and placement of task instructions on problem-solving creativity. The Journal of Creative Behavior, 52(4), 335-353. Eastman, C. M. (1969). Cognitive processes and ill-defined problems: a case study from design. In Proceedings of the first joint international conference on I.A. (pp. 669-690).

Epskamp, S. (2019). semPlot: Path diagrams and visual analysis of various SEM packages' output. Available at https://CRAN.R-project.org/package=semPlot 
Epskamp, S., Borsboom, D., \& Fried, E. I. (2018). Estimating psychological networks and their accuracy: A tutorial paper. Behavior Research Methods, 50(1), 195-212. https://doi.org/10.3758/s13428-017-0862-1

Epskamp, S., Cramer, A. O. J., Waldorp, L. J., Schmittmann, V. D., \& Borsboom, D. (2012). qgraph: Network visualizations of relationships in psychometric data. Journal of Statistical Software, 48(4), 1-18.

Flake, J. K., Pek, J., \& Hehman, E. (2017). Construct validation in social and personality research: Current practice and recommendations. Social Psychological and Personality Science, 8(4), 370-378. https://doi.org/10.1177/1948550617693063

Forthmann, B., Gerwig, A., Holling, H., Çelik, P., Storme, M., \& Lubart, T. (2016). The becreative effect in divergent thinking: The interplay of instruction and object frequency. Intelligence, 57, 25-32.

Sullivan, D. M., \& Ford, C. M. (2010). The alignment of measures and constructs in organizational research: The case of testing measurement models of creativity. Journal of Business and Psychology, 25(3), 505-521. https://doi.org/10.1007/s10869-009-9147-8

Glăveanu, V. P. (2013). Rewriting the language of creativity: The Five A's framework. Review of General Psychology, 17(1), 69-81. https://doi.org/10.1037/a0029528

Horn, D., \& Salvendy, G. (2006a). Product creativity: Conceptual model, measurement and characteristics. Theoretical Issues in Ergonomics Science, 7(4), 395-412. https://doi.org/10.1080/14639220500078195

Horn, D., \& Salvendy, G. (2006b). Consumer-based assessment of product creativity: A review and reappraisal. Human Factors and Ergonomics in Manufacturing, 16(2), 155-175. $\underline{\text { https://doi.org/10.1002/hfm.20047 }}$ 
Horn, D., \& Salvendy, G. (2009). Measuring consumer perception of product creativity: Impact on satisfaction and purchasability. Human Factors and Ergonomics in Manufacturing \& Service Industries, 19(3), 223-240. https://doi.org/10.1002/hfm.20150

James, G., Witten, D., Hastie, T., \& Tibshirani, A. (2014). An introduction to statistical learning: With applications in R. New York, (NY): Springer Publishing.

Janssen, P., Jégou, P., Nouguier, B., \& Vilarem, M. C. (1989). A filtering process for general constraint-satisfaction problems: Achieving pairwise-consistency using an associated binary representation. In IEEE International Workshop on Tools for Artificial Intelligence (pp. 420421).

Koo T.K., Li M.Y. (2016) A guideline of selecting and reporting intraclass correlation coefficients for reliability research. J Chiropr Med 15:155-163.

\section{https://doi.org/10.1016/i.jcm.2016.02.012}

Kudrowitz, B. M., \& Wallace, D. (2013). Assessing the quality of ideas from prolific, early-stage product ideation. Journal of Engineering Design, 24(2), 120-139.

https://doi.org/10.1080/09544828.2012.676633

Lonergan, D. C., Scott, G. M., \& Mumford, M. D. (2004). Evaluative aspects of creative thought: Effects of appraisal and revision standards. Creativity Research Journal, 16(2-3), 231-246.

Lubart, T. (2017). The 7 C's of creativity. The Journal of Creative Behavior, 51(4), 293-296. https://doi.org/10.1002/jocb.190

MacCrimmon, K. R., \& Wagner, C. (1994). Stimulating ideas through creative software. Management Science, 40(11), 1514-1532. https://doi.org/10.1287/mnsc.40.11.1514

Moran, S. (2010). The roles of creativity in society. In J. C. Kaufman \& R. J. Sternberg (Eds.), The Cambridge handbook of creativity (pp. 74-90). Cambridge University Press. 
Mulet, E., Royo, M., Chulvi, V., \& Galán, J. (2017). Relationship between the degree of creativity and the quality of design outcomes. DYNA, 84(200), 38-45. https://doi.org/10.15446/dyna.v84n200.53582

Mumford, M. D., Baughman, W. A., Maher, M. A., Costanza, D. P., \& Supinski, E. P. (1997). Processbased measures of creative problem-solving skills: IV. Category combination. Creativity Research Journal, 10(1), 59-71. https://doi.org/10.1207/s15326934crj1001_ 7

O’Quin, K., \& Besemer, S. P. (1989). The development, reliability, and validity of the revised creative product semantic scale. Creativity Research Journal, 2(4), 267-278. https://doi.org/10.1080/10400418909534323

Pichot, N., Bonetto, E., Arciszewski, T., Bonnardel, N., \& Pavani, J.-B. (2020, September 16). Towards a general factor of Disruptivity: The most novel creative objects tend to be the least valuable and feasible ones. Retrieved from osf.io/4fq3a

Plucker, J. A., \& Renzulli, J. S. (1999). Psychometric approaches to the study of human creativity. In Handbook of creativity (pp. 35-61). Cambridge University Press.

Potter, R. E., \& Balthazard, P. (2004). The role of individual memory and attention processes during electronic brainstorming. MIS Quarterly, 28(4), 621-643.

R Core Team (2019). R: A language and environment for statistical computing. Vienna: $\mathrm{R}$ Foundation for Statistical Computing.

Reiter-Palmon, R., \& Arreola, N. J. (2015). Does generating multiple ideas lead to increased creativity? A comparison of generating one idea vs. many. Creativity Research Journal, 27(4), 369-374.

Reiter-Palmon, R., Mumford, M. D., O'Connor Boes, J., \& Runco, M. A. (1997). Problem construction and creativity: The role of ability, cue consistency, and active processing. Creativity Research Journal, 10(1), 9-23.

https://doi.org/10.1207/s15326934crj1001_2 
Revelle, W. (2019). psych: Procedures for psychological, psychometric, and personality research. Northwestern University.

Rhodes, M. (1961). An analysis of creativity. The Phi Delta Kappan, 42(7), 305-310.

Rietzschel, E. F., Nijstad, B. A., \& Stroebe, W. (2010). The selection of creative ideas after individual idea generation: Choosing between creativity and impact. British Journal of Psychology, 101(1), 47-68. https://doi.org/10.1348/000712609X414204

Rosseel, Y. (2012). lavaan: An R package for structural equation modeling. Journal of Statistical Software, 48(2), 1-36.

Runco, M. A., \& Charles, R. E. (1993). Judgments of originality and appropriateness as predictors of creativity. Personality and Individual Differences, 15(5), 537-546.

https://doi.org/10.1016/0191-8869(93)90337-3

Runco, M. A., \& Jaeger, G. J. (2012). The standard definition of creativity. Creativity Research Journal, 24(1), 92-96. https://doi.org/10.1080/10400419.2012.650092

Sarkar, P., \& Chakrabarti, A. (2011). Assessing design creativity. Design Studies, 32(4), 348-383. https://doi.org/10.1016/j.destud.2011.01.002

Simmering, M. J., Fuller, C. M., Richardson, H. A., Ocal, Y., \& Atinc, G. M. (2015). Marker variable choice, reporting, and interpretation in the detection of common method variance: A review and demonstration. Organizational Research Methods, 18(3), 473-

511. https://doi.org/10.1177/1094428114560023

Simon, H. A. (1973). The structure of ill structured problems. Artificial Intelligence, 4, 181-201.

Watts, L. L., Steele, L. M., Medeiros, K. E., \& Mumford, M. D. (2019). Minding the gap between generation and implementation: Effects of idea source, goals, and climate on selecting and refining creative ideas. Psychology of Aesthetics, Creativity, and the Arts, 13(1), 2. https://doi.org/10.1037/aca0000157 
Williams, L. J., Hartman, N., \& Cavazotte, F. (2010). Method variance and marker variables: A review and comprehensive CFA marker technique. Organizational Research Methods, 13(3), 477-514. https://doi.org/10.1177/1094428110366036

Weisberg, R. W. (2015). On the usefulness of "value" in the definition of creativity. Creativity Research Journal, 27(2), 111-124. https://doi.org/10.1080/10400419.2015.1030320

Weisberg, R. W. (2018). Response to Harrington on the definition of creativity, Creativity Research Journal, 30(4), 461-465

White, A., \& Smith, B. L. (2001). Assessing advertising creativity using the Creative Product Semantic Scale. Journal of Advertising Research, 41(6), 27-34. https://doi.org/10.2501/JAR$\underline{41-6-27-34}$ 